FACTA UNIVERSITATIS (NIŠ)

Ser. Math. Inform. Vol. 34, No 4 (2019), 689-708

https://doi.org/10.22190/FUMI1904689A

\title{
HYPERHROUPS AND $H_{v}$-GROUPS ASSOCIATED TO ELEMENTS WITH FOUR OXIDATION STATES
}

\author{
Rawia Al-Jinani, Madeleine Al-Tahan and Bijan Davvaz
}

(C) 2019 by University of Niš, Serbia | Creative Commons Licence: CC BY-NC-ND

\begin{abstract}
The theory of hyperstructures is of great importance due to its connections to various fields of Science. $H_{v}$-structures are hyperstructures where the equality is replaced by the nonempty intersection. This class of the hyperstructures is very large so one can use it in order to define several objects that they are not possible to be defined in the classical hyperstructure theory. This paper attempts an exposition of the connection between hyperstructure $\left(H_{v}\right.$-structure) theory and certain type of chemical reactions. In this regard, we consider elements with four oxidation states and investigate their mathematical structures.
\end{abstract}

Keywords. Hyperstructures; chemical reaction; mathematical structure; chemical reaction.

\section{Introduction}

The origin of hypergroups can be followed back to Marty [18] who introduced it in 1934 at the eighth Congress of Scandinavian Mathematicians. He generalized the concept of a group, where the theory of groups is the oldest branch of ordinary algebra, by considering the result of the "interaction" between two elements of a non-empty set to be a non empty set of elements. He published some notes on hypergroups, using them in different contexts as algebraic functions, rational fractions, non-commutative groups. The theory knew an important progress starting with the 70's, when its research area has enlarged and new concepts were introduced and studies such as canonical hypergroups, hyperrings, hypermodules, etc. A generalization of algebraic hyperstructures was introduced in 1990 by T. Vougiouklis [21] where he defined weak hyperstructures. Many researchers such as Corsini [6], Corsini and Leoreanu [7], Davvaz [8, 9], Davvaz and Leoreanu-Fotea [16], and Vougiouklis [20] wrote books related to hyperstructure theory and their applications.

Received March 17, 2019; accepted July 22, 2019

2010 Mathematics Subject Classification. Primary 20N20 
One of motivations for the study of hyperstructures comes from chemical reactions. In [11], Davvaz and Dehghan-Nezhad provided examples of hyperstructures associated with chain reactions. In [13], Davvaz et al. introduced examples of weak hyperstructures associated with dismutation reactions. In [15], Davvaz et al. investigated the examples of hyperstructures and weak hyperstructures associated with redox reactions. In [1], Al-Tahan et al. presented three different examples of weak hyperstructures associated to elechtrochemical cells. In [5]. Chung et al. investigated mathematical structures of chemical reactions for three consecutive oxidation states of elements. Some authors considered particular elements with four oxidation states and investigated their chemical hyperstructures. For example, Chun in [4] presented chemical hyperstructures of chemical reactions for a set of Titanium and Al-Tahan et al. in [2] studied chemical hyperstructures of chemical reactions for a set of Astatine, a set of Tellurium and a set of Bismuth. Then in [3], they studied mathematical structures of chemical reactions for arbitrary elements with four oxidation states.

In this paper, we consider an arbitrary element with four oxidation states and investigate its algebraic hyperstructures and it is organized as follows: After an Introduction, Section 2 presents some definitions and concepts related to (weak) hyperstructures that are used throughout the paper. Section 3 presents chemical hyperstructures using redox reactions of an arbitrary element with four oxidation states as algebraic hyperstructures under certain conditions. Finally, Section 4 presents some examples of elements with four oxidation states that satisfy the conditions presented in Section 3.

\section{Preliminaries}

In this section, we present some definitions and concepts related to (weak) hyperstructures that are used throughout the paper.

Definition 2.1. [8] Let $H$ be a non-empty set. Then, a mapping $\circ: H \times H \rightarrow$ $\mathcal{P}^{*}(H)$ is called a binary hyperoperation on $H$, where $\mathcal{P}^{*}(H)$ is the family of all non-empty subsets of $H$. The couple $(H, \circ)$ is called a hypergroupoid.

In the above definition, if $A$ and $B$ are two non-empty subsets of $H$ and $x \in H$, then we define:

$$
A \circ B=\bigcup_{\substack{a \in A \\ b \in B}} a \circ b, x \circ A=\{x\} \circ A \text { and } A \circ x=A \circ\{x\} .
$$

$H_{v}$-structures were introduced by $\mathrm{T}$. Vougiouklis as a generalization of the wellknown algebraic hyperstructures [19, 21], also see [12, 20]. Some axioms of classical algebraic hyperstructures are replaced by their corresponding weak axioms in $H_{v^{-}}$ structures. Most of $H_{v}$-structures are used in representation theory. 
Definition 2.2. $[20]$ A hypergroupoid $(H, \circ)$ is called an $H_{v}$-semigroup if $(x \circ(y \circ$ $z)) \cap((x \circ y) \circ z) \neq \varnothing$ for all $x, y, z \in H$.

A subset $K$ of an $H_{v}$-semigroup is an $H_{v}$-subsemigroup if $K$ is an $H_{v}$-semigroup. An element $x \in H$ is called idempotent if $x^{2}=x \circ x=x$ and an element $e \in H$ is called an identity of $(H, \circ)$ if $x \in x \circ e \cap e \circ x$, for all $x \in H$. The hypergroupoid $(H, \circ)$ is said to be commutative if $x \circ y=y \circ x$, for all $x, y \in H$.

We present an example of a commutative $H_{v}$-semigroup.

Example 2.1. [2] Let $H=\{a, b, c, d\}$ and define $(H, \star)$ by the following table:

\begin{tabular}{|c|c|c|c|c|}
\hline$\star$ & $a$ & $b$ & $c$ & $d$ \\
\hline$a$ & $a$ & $\{a, c\}$ & $\{a, c\}$ & $c$ \\
\hline$b$ & $\{a, c\}$ & $\{a, c\}$ & $\{a, c\}$ & $c$ \\
\hline$c$ & $\{a, c\}$ & $\{a, c\}$ & $c$ & $\{c, d\}$ \\
\hline$d$ & $c$ & $c$ & $\{c, d\}$ & $d$ \\
\hline
\end{tabular}

Then $(H, \star)$ is a commutative $H_{v}$-semigroup.

Definition 2.3. [6] A hypergroupoid $(H, \circ)$ is called a:

1. semihypergroup if for every $x, y, z \in H$, we have $x \circ(y \circ z)=(x \circ y) \circ z$;

2. quasi-hypergroup if for every $x \in H, x \circ H=H=H \circ x$ (The latter condition is called the reproduction axiom);

3. hypergroup if it is a semihypergroup and a quasi-hypergroup.

We present an example of a commutative hypergroup of four elements.

Example 2.2. [2] Let $H=\{a, b, c, d\}$ and define "o" on $H$ by the following table:

\begin{tabular}{|c|c|c|c|c|}
\hline$\circ$ & $a$ & $b$ & $c$ & $d$ \\
\hline$a$ & $a$ & $\{a, b, c\}$ & $\{a, c\}$ & $H$ \\
\hline$b$ & $\{a, b, c\}$ & $\{a, b, c\}$ & $\{a, b, c\}$ & $H$ \\
\hline$c$ & $\{a, c\}$ & $\{a, b, c\}$ & $c$ & $\{c, d\}$ \\
\hline$d$ & $H$ & $H$ & $\{c, d\}$ & $d$ \\
\hline
\end{tabular}

Then $(H, \circ)$ is a commutative hypergroup.

Definition 2.4. [6] Two hypergroupoids $(H, \circ)$ and $(K, \star)$ are said to be isomorphic hypergroupoids, written as $H \cong K$, if there exists a bijective function $f: H \rightarrow K$ such that $f(x \circ y)=f(x) \star f(y)$ for all $x, y \in H$. 


\section{Chemical hyperstructures for elements with four oxidation states}

Through the Latimer diagrams of all elements, we selected a lot of chemical species that were recorded four consecutive standard reduction potentials in acidic and/or basic solution [17].

Example 3.1. [17] The following Latimer diagram are for Astatine, Copper, Iridium, and Carbon respectively that are elements with four consecutive standard reduction potentials in acidic and/or basic solution.

$$
\begin{gathered}
\mathrm{At0}_{3}^{-} \longrightarrow_{0.5} \mathrm{At0}^{-} \longrightarrow_{0} \mathrm{At}_{2} \longrightarrow_{0.2} \mathrm{At}^{-} ; \\
\mathrm{CuO}^{+} \longrightarrow_{1.8} \mathrm{Cu}^{2+} \longrightarrow_{0.159} \mathrm{Cu}^{+} \longrightarrow_{0.521} \mathrm{Cu} \\
\mathrm{Ir}^{6+} \longrightarrow_{0.4} \mathrm{Ir}^{4+} \longrightarrow_{0.1} \mathrm{Ir}^{3+} \longrightarrow_{0.1} \mathrm{Ir} ; \\
\mathrm{C}^{6+} \longrightarrow_{-1.01} \mathrm{C}^{+} \longrightarrow_{-0.52} \mathrm{C} \longrightarrow_{-0.7} \mathrm{C}^{4-} .
\end{gathered}
$$

Let $A, B, C$, and $D$ be chemical species of an arbitrary element $\mathrm{S}$ and let $n_{1}$ be the difference of the oxidation number between $D$ and $C, n_{2}$ be the difference of the oxidation number between $C$ and $B$ and $n_{3}$ be the difference of the oxidation number between $B$ and $A$. Let $\alpha, \beta$, and $\gamma$ be the potential difference between $D, C, B$, and $A$ like in the following Latimer diagram of $S$.

$$
D \longrightarrow_{\alpha} C \longrightarrow_{\beta} B \longrightarrow_{\gamma} A .
$$

We present the following reductions.

(1) $D \longrightarrow B, E_{1}=\frac{\alpha n_{1}+\beta n_{2}}{n_{1}+n_{2}}$,

(2) $D \longrightarrow A, E_{2}=\frac{\alpha n_{1}+\beta n_{2}+\gamma n_{3}}{n_{1}+n_{2}+n_{3}}$,

(3) $C \longrightarrow A, E_{3}=\frac{\beta n_{2}+\gamma n_{3}}{n_{2}+n_{3}}$.

Let $\left\{x, x^{\prime}, y, y^{\prime}\right\} \subseteq\{A, B, C, D\}$ such that $x \longrightarrow_{a} x^{\prime}$ and $y \longrightarrow_{b} y^{\prime}$ where $a, b$ are potential differences. We get the following redox reaction:

$$
x+x^{\prime} \longrightarrow_{a+b} y+y^{\prime}
$$

If $E=a+b>0$ then our redox reaction is spontaneous. Otherwise it is not spontaneous.

In this paper, we are concerned about the chemical hyperstructures of $S$ under the condition $\alpha \geq \gamma \geq \beta$. We consider the four different cases, case $\alpha=\gamma=\beta$, case $\alpha>\gamma>\beta$, case $\alpha>\gamma=\beta$, and case $\alpha=\gamma>\beta$.In this regard, we consider each case separately and find the (weak) hyperstructures associated to it.

\section{1. $\quad$ Case $\alpha=\gamma=\beta$}

Let $H=\{A, B, C, D\}$, it is clear that: 


$$
E_{1}=E_{2}=E_{3}=\alpha=\beta=\gamma .
$$

For all $x, y \in H$, we define " $\oplus$ " on $H$ as follows: $x \oplus y=z$ where $z$ in the product of the spontaneous redox reaction with greatest potential difference that occurs between $x$ and $y$.

Then we obtain the following table for $(H, \oplus)$.

\begin{tabular}{|c|c|c|c|c|}
\hline$\oplus$ & $A$ & $B$ & $C$ & $D$ \\
\hline $\mathrm{A}$ & $\mathrm{A}$ & $\{\mathrm{A}, \mathrm{B}\}$ & $\{\mathrm{A}, \mathrm{C}\}$ & $\{\mathrm{A}, \mathrm{D}\}$ \\
\hline $\mathrm{B}$ & $\{\mathrm{A}, \mathrm{B}\}$ & $\mathrm{B}$ & $\{\mathrm{B}, \mathrm{C}\}$ & $\{\mathrm{B}, \mathrm{D}\}$ \\
\hline $\mathrm{C}$ & $\{\mathrm{A}, \mathrm{C}\}$ & $\{\mathrm{B}, \mathrm{C}\}$ & $\mathrm{C}$ & $\{\mathrm{C}, \mathrm{D}\}$ \\
\hline $\mathrm{D}$ & $\{\mathrm{A}, \mathrm{D}\}$ & $\{\mathrm{B}, \mathrm{D}\}$ & $\{\mathrm{C}, \mathrm{D}\}$ & $\mathrm{D}$ \\
\hline
\end{tabular}

Theorem 3.1. Let $H=\{A, B, C, D\}$. Then $(H, \oplus)$ is a commutative hypergroup.

Proof. The proof is straightforward since $(H, \oplus)$ is the Biset hypergroup with four elements.

\section{2. $\quad$ Case $\alpha>\gamma>\beta$}

Let $H=\{A, B, C, D\}$, it is clear that:

$$
\beta<E_{1}<\alpha, \beta<E_{2}<\alpha, \beta<E_{3}<\gamma .
$$

The following are all possible spontaneous redox combinations for $H$.

$A+A \longrightarrow A+A[0]$

For the spontaneous reactions of $A+B$, we consider the three cases: Case $E_{2}<\gamma$, Case $E_{2}>\gamma$ and Case $E_{2}=\gamma$.

Case $E_{2}<\gamma$. We get that

$$
A+B \longrightarrow\left\{\begin{array}{c}
C+A\left[\gamma-E_{3}\right] \\
D+A\left[\gamma-E_{2}\right] \\
B+A[0]
\end{array}\right.
$$

Case $E_{2}>\gamma$. We get that

$$
A+B \longrightarrow\left\{\begin{array}{c}
C+A\left[\gamma-E_{3}\right] \\
B+A[0]
\end{array}\right.
$$

Case $E_{2}=\gamma$. We get that

$$
A+B \longrightarrow\left\{\begin{array}{c}
C+A\left[\gamma-E_{3}\right] \\
B+A[0]
\end{array}\right.
$$

$A+C \longrightarrow C+A[0]$

For the spontaneous reaction $A+D$, we consider the 6 cases: Case $E_{2}, E_{1}<\gamma$, 
Case $E_{2}, E_{1}>\gamma$, Case $E_{2}, E_{1}=\gamma$, Case $E_{1}>\gamma, E_{2}<\gamma$, Case $E_{1}>\gamma, E_{2}=\gamma$, Case $E_{1}<\gamma, E_{2}>\gamma$, Case $E_{1}<\gamma, E_{2}=\gamma$.

Case $E_{2}, E_{1}<\gamma$. We get that

$$
A+D \longrightarrow\left\{\begin{array}{c}
B+C[\alpha-\gamma] \\
C+C\left[\alpha-E_{3}\right] \\
D+C\left[\alpha-E_{2}\right] \\
D+A[0]
\end{array}\right.
$$

Case $E_{2}, E_{1}>\gamma$. We get that

$$
A+D \longrightarrow\left\{\begin{array}{c}
B+C[\alpha-\gamma] \\
B+B\left[E_{1}-\gamma\right] \\
B+A\left[E_{2}-\gamma\right] \\
C+C\left[\alpha-E_{3}\right] \\
D+C\left[\alpha-E_{2}\right] \\
D+A[0]
\end{array}\right.
$$

Case $E_{1}=\gamma, E_{2}=\gamma$. We get that

$$
A+D \longrightarrow\left\{\begin{array}{c}
B+C[\alpha-\gamma] \\
C+C\left[\alpha-E_{3}\right] \\
C+B\left[E_{1}-E_{3}\right] \\
C+A\left[E_{2}-E_{3}\right] \\
D+C\left[\alpha-E_{2}\right] \\
D+A[0]
\end{array}\right.
$$

Case $E_{1}>\gamma, E_{2}<\gamma$. We get that

$$
A+D \longrightarrow\left\{\begin{array}{c}
B+C[\alpha-\gamma] \\
B+B\left[E_{1}-\gamma\right] \\
C+C\left[\alpha-E_{3}\right] \\
D+C\left[\alpha-E_{2}\right] \\
D+A[0]
\end{array}\right.
$$

Case $E_{1}>\gamma, E_{2}=\gamma$. We get that

$$
A+D \longrightarrow\left\{\begin{array}{c}
B+C[\alpha-\gamma] \\
B+B\left[E_{1}-\gamma\right] \\
C+C\left[\alpha-E_{3}\right] \\
C+A\left[\gamma-E_{3}\right] \\
D+C[\alpha-\gamma] \\
D+A[0]
\end{array}\right.
$$

Case $E_{1}<\gamma, E_{2}>\gamma$. We get that

$$
A+D \longrightarrow\left\{\begin{array}{c}
B+C[\alpha-\gamma] \\
B+A\left[E_{2}-\gamma\right] \\
C+C\left[\alpha-E_{3}\right] \\
D+C\left[\alpha-E_{2}\right] \\
D+A[0]
\end{array}\right.
$$


Case $E_{1}<\gamma, E_{2}=\gamma$. We get that

$$
A+D \longrightarrow\left\{\begin{array}{c}
B+C[\alpha-\gamma] \\
C+C\left[\alpha-E_{3}\right] \\
C+A\left[\gamma-E_{3}\right] \\
D+C\left[\alpha-E_{2}\right] \\
D+B\left[E_{1}-\gamma\right] \\
D+A[0]
\end{array}\right.
$$

For the spontaneous reaction $B+B$, we consider the three cases: Case $E_{1}<\gamma$, Case $E_{1}>\gamma$ and Case $E_{1}=\gamma$.

Case $E_{1}<\gamma$. We get that

$$
B+B \longrightarrow\left\{\begin{array}{c}
B+B[0] \\
A+C[-\beta+\gamma] \\
A+D\left[\gamma-E_{1}\right]
\end{array}\right.
$$

Case $E_{1}>\gamma$. We get that

$$
B+B \longrightarrow\left\{\begin{array}{c}
B+B[0] \\
A+C[-\beta+\gamma]
\end{array}\right.
$$

Case $E_{1}=\gamma$. We get that

$$
\begin{gathered}
B+B \longrightarrow\left\{\begin{array}{c}
B+B[0] \\
A+C[-\beta+\gamma]
\end{array}\right. \\
B+C \longrightarrow\left\{\begin{array}{c}
C+B[0] \\
C+A\left[-\beta+E_{3}\right]
\end{array}\right. \\
B+D \longrightarrow\left\{\begin{array}{c}
C+C[-\beta+\alpha] \\
C+B\left[-\beta+E_{1}\right] \\
C+A\left[-\beta+E_{2}\right] \\
D+C\left[\alpha-E_{1}\right] \\
D+B[0]
\end{array}\right.
\end{gathered}
$$

$C+C \longrightarrow C+C[0]$

$C+D \longrightarrow D+C[0]$

$D+D \longrightarrow D+D[0]$

Remark 3.1. For all $x, y \in H$, the major product of $x$ and $y$ is that with the greatest potential difference.

For all $x, y \in H$, we define " $\oplus_{i}$ " with $i=1,2, \ldots, 7$ on $H$ for $E_{2}, E_{1}<\gamma$, Case $E_{2}, E_{1}>\gamma$, Case $E_{2}, E_{1}=\gamma$, Case $E_{1}>\gamma, E_{2}<\gamma$, Case $E_{1}>\gamma, E_{2}=\gamma$, Case $E_{1}<\gamma, E_{2}>\gamma$, Case $E_{1}<\gamma, E_{2}=\gamma$. Where $x \oplus_{i} y=z_{i}$ and $z_{i}$ is in the product of the spontaneous redox reaction with greatest potential difference that occurs between $x$ and $y$.

Since the major part for all cases of $E_{1}, E_{2}$ under the case $\alpha>\gamma>\beta$ is the same, we get $\left(H, \oplus_{i}\right)=\left(H, \oplus_{1}\right)$ for $i=1,2, \ldots, 7$. 


\begin{tabular}{|c|c|c|c|c|}
\hline$\oplus_{1}$ & $A$ & $B$ & $C$ & $D$ \\
\hline$A$ & $A$ & $\{A, C\}$ & $\{A, C\}$ & $C$ \\
\hline$B$ & $\{A, C\}$ & $\{A, C\}$ & $\{A, C\}$ & $C$ \\
\hline$C$ & $\{A, C\}$ & $\{A, C\}$ & $C$ & $\{C, D\}$ \\
\hline$D$ & $C$ & $C$ & $\{C, D\}$ & $D$ \\
\hline
\end{tabular}

Theorem 3.2. $\left(H, \oplus_{1}\right)$ is commutative $H_{v}$-semigroup.

Proof. $\left(H, \oplus_{1}\right)$ is isomorphic to the $H_{v}$-semigroup $(H, \star)$ presented in Example 2.1.

Let $H=\{A, B, C, D\}$, for all $x, y \in H$, we define " $\otimes_{1}$ " for the case $E_{1}, E_{2}<\gamma$, " $\otimes_{2}$ " for the case $E_{1}, E_{2}>\gamma$, " $\otimes_{3}$ " for the case $E_{1}<\gamma, E_{2}>\gamma$, " $\otimes_{4}$ " for the case $E_{1}>\gamma, E_{2}<\gamma, " \otimes_{5}$ " for the case $E_{1}<\gamma, E_{2}=\gamma$, " $\otimes_{6}$ " for the case $E_{1}>\gamma, E_{2}=\gamma$ and " $\otimes_{7}$ " for the case $E_{1}=E_{2}=\gamma$. Here, $x \otimes_{i} y=z$, where $z$ is in the product of any spontaneous redox reaction that occurs between $x$ and $y$ for $i=1,2, \ldots, 7$.

Remark 3.2. $\left(H, \otimes_{2}\right)=\left(H, \otimes_{6}\right)=\left(H, \otimes_{7}\right)$ and $\left(H, \otimes_{3}\right)=\left(H, \otimes_{5}\right)$.

$\left(H, \otimes_{i}\right)$ for $i=1,2, \ldots, 7$ are given by tables $3.1,3.2,3.3,3.4$.

Table 3.1: $\left(H, \otimes_{1}\right)$

\begin{tabular}{|c|c|c|c|c|}
\hline$\otimes_{1}$ & $A$ & $B$ & $C$ & $D$ \\
\hline$A$ & $A$ & $H$ & $\{A, C\}$ & $H$ \\
\hline$B$ & $H$ & $H$ & $\{A, B, C\}$ & $H$ \\
\hline$C$ & $\{A, C\}$ & $\{A, B, C\}$ & $C$ & $\{C, D\}$ \\
\hline$D$ & $H$ & $H$ & $\{C, D\}$ & $D$ \\
\hline
\end{tabular}

Table 3.2: $\left(H, \otimes_{2}\right)$

\begin{tabular}{|c|c|c|c|c|}
\hline$\otimes_{2}$ & $A$ & $B$ & $C$ & $D$ \\
\hline$A$ & $A$ & $\{A, B, C\}$ & $\{A, C\}$ & $H$ \\
\hline$B$ & $\{A, B, C\}$ & $\{A, B, C\}$ & $\{A, B, C\}$ & $H$ \\
\hline$C$ & $\{A, C\}$ & $\{A, B, C\}$ & $C$ & $\{C, D\}$ \\
\hline$D$ & $H$ & $H$ & $\{C, D\}$ & $D$ \\
\hline
\end{tabular}


Table 3.3: $\left(H, \otimes_{3}\right)$

\begin{tabular}{|c|c|c|c|c|}
\hline$\otimes_{3}$ & $A$ & $B$ & $C$ & $D$ \\
\hline$A$ & $A$ & $\{A, B, C\}$ & $\{A, C\}$ & $H$ \\
\hline$B$ & $\{A, B, C\}$ & $H$ & $\{A, B, C\}$ & $H$ \\
\hline$C$ & $\{A, C\}$ & $\{A, B, C\}$ & $C$ & $\{C, D\}$ \\
\hline$D$ & $H$ & $H$ & $\{C, D\}$ & $D$ \\
\hline
\end{tabular}

Table 3.4: $\left(H, \otimes_{4}\right)$

\begin{tabular}{|c|c|c|c|c|}
\hline$\otimes_{4}$ & $A$ & $B$ & $C$ & $D$ \\
\hline$A$ & $A$ & $H$ & $\{A, C\}$ & $H$ \\
\hline$B$ & $H$ & $\{A, B, C\}$ & $\{A, B, C\}$ & $H$ \\
\hline$C$ & $\{A, C\}$ & $\{A, B, C\}$ & $C$ & $\{C, D\}$ \\
\hline$D$ & $H$ & $H$ & $\{C, D\}$ & $D$ \\
\hline
\end{tabular}

Remark 3.3. It is clear that $\left(H, \otimes_{i}\right), i=1,2,3,4$ are not isomorphic.

Proposition 3.1. $\left(H, \otimes_{1}\right)$ is a commutative quasi-hypergroup.

Proof. Since all elements of $H$ are present in every row and column, it follows that $\left(H, \otimes_{1}\right)$ is a quasi-hypergroup.

Proposition 3.2. $\left(\{A, C\}, \otimes_{1}\right)$ and $\left(\{C, D\}, \otimes_{1}\right)$ are commutative semihypergroups.

Proof. The proof is straightforward.

Proposition 3.3. $\left(H, \otimes_{1}\right)$ is a commutative semihypergroup.

Proof. Since $\left(\{A, C\}, \otimes_{1}\right)$ and $\left(\{C, D\}, \otimes_{1}\right)$ are hypergroups, it suffices to consider the following cases for associativity:

$A \otimes_{1}\left(D \otimes_{1} z\right)=\left(A \otimes_{1} D\right) \otimes_{1} z=H$.

$A \otimes_{1}\left(B \otimes_{1} z\right)=\left(A \otimes_{1} B\right) \otimes_{1} z=H$.

$A \otimes_{1}\left(C \otimes_{1} z\right)=\left(A \otimes_{1} C\right) \otimes_{1} z=H$ for all $z \in H-\{C\}$.

$A \otimes_{1}\left(C \otimes_{1} C\right)=\left(A \otimes_{1} C\right) \otimes_{1} C=\{A, C\}$.

$B \otimes_{1}\left(y \otimes_{1} z\right)=\left\{\begin{array}{ll}\{A, B, C\} & \text { if } y=C, z=C \\ H, & \text { otherwise }\end{array}=\left(B \otimes_{1} y\right) \otimes_{1} z\right.$.

$C \otimes_{1}\left(y \otimes_{1} B\right)=\left\{\begin{array}{cc}\{A, B, C\} & \text { if } y=C \\ H, & \text { otherwise }\end{array}=\left(C \otimes_{1} y\right) \otimes_{1} B\right.$.

$C \otimes_{1}\left(B \otimes_{1} z\right)=\left\{\begin{array}{cc}\{A, B, C\} & \text { if } z=C \\ H & \text { otherwise }\end{array}=\left(C \otimes_{1} B\right) \otimes_{1} z\right.$. 
$D \otimes_{1}\left(y \otimes_{1} z\right)=\left(D \otimes_{1} y\right) \otimes_{1} z=G$ for all $y, z \notin\{C, D\}$. Thus, $\left(H, \otimes_{1}\right)$ is a semihypergroup..

Theorem 3.3. $\left(H, \otimes_{1}\right)$ is a commutative hypergroup.

Proof. The proof is follows from Propositions 3.1 and 3.3.

Theorem 3.4. $\left(H, \otimes_{i}\right)$ for $i=2,3,5,6,7$ are commutative hypergroups.

Proof. By following the same proof done in Theorem 3.3, we get that $\left(H, \otimes_{i}\right)$ for $i=2,3,5,6,7$ are commutative hypergroups.

Theorem 3.5. $\left(H, \otimes_{4}\right)$ is a commutative $H_{v}$-group.

Proof. Easy computations show that $\left(H, \otimes_{4}\right)$ is a commutative $H_{v}$-group.

\subsection{Case $\alpha>\gamma=\beta$}

Let $H=\{A, B, C, D\}$, it is clear that:

$$
\beta=\gamma<E_{1}<\alpha, \beta=\gamma<E_{2}<\alpha, \beta=E_{3}=\gamma .
$$

The following are all possible spontaneous redox combinations for $H$.

$A+A \longrightarrow A+A[0]$

$A+B \longrightarrow B+A[0]$

$A+C \longrightarrow C+A[0]$

$A+D \longrightarrow\left\{\begin{array}{c}B+C[\alpha-\gamma] \\ B+B\left[E_{1}-\gamma\right] \\ B+A\left[E_{2}-\gamma\right] \\ C+C\left[\alpha-E_{3}\right] \\ D+C\left[\alpha-E_{2}\right] \\ D+A[0]\end{array}\right.$

$B+B \longrightarrow B+B[0]$

$B+C \longrightarrow C+B[0]$

$B+D \longrightarrow\left\{\begin{array}{c}C+C[-\beta+\alpha] \\ C+B\left[-\beta+E_{1}\right] \\ C+A\left[\beta-E_{2}\right] \\ D+C\left[\alpha-E_{1}\right] \\ D+B[0]\end{array}\right.$

$C+C \longrightarrow C+C[0]$

$C+D \longrightarrow D+C[0]$

$D+D \longrightarrow D+D[0]$

For all $x, y \in H$, we define " $\oplus_{8}$ " on $H$ as follows: $x \oplus_{8} y=z$, where $z$ is the major product of the spontaneous redox reaction that occurs between $x$ and $y$.

We obtain the following table for $\left(H, \oplus_{8}\right)$ : 


\begin{tabular}{|c|c|c|c|c|}
\hline$\oplus_{8}$ & $A$ & $B$ & $C$ & $D$ \\
\hline$A$ & $A$ & $\{A, B\}$ & $\{A, C\}$ & $\{B, C\}$ \\
\hline$B$ & $\{A, B\}$ & $B$ & $\{B, C\}$ & $C$ \\
\hline$C$ & $\{A, C\}$ & $\{B, C\}$ & $C$ & $\{C, D\}$ \\
\hline$D$ & $\{B, C\}$ & $C$ & $\{C, D\}$ & $D$ \\
\hline
\end{tabular}

Proposition 3.4. Let $H=\{A, B, C, D\}$. Then $\left(H, \oplus_{8}\right)$ is a commutative $H_{v^{-}}$ semigroup.

Proof. Let $x, y, z \in H$. We consider the following cases for $x, y, z$.

- If $x=y=z$ then $x \in x \oplus_{8}\left(y \oplus_{8} z\right) \cap\left(x \oplus_{8} y\right) \oplus_{8} z$.

- If $x=A$ then $A \in x \oplus_{8}\left(y \oplus_{8} z\right) \cap\left(x \oplus_{8} y\right) \oplus_{8} z$ for all $(y, z) \neq(D, D)$ and $C \in x \oplus_{8}\left(D \oplus_{8} D\right) \cap\left(x \oplus_{8} D\right) \oplus_{8} D$.

- If $x=B$ then $B \in x \oplus_{8}\left(y \oplus_{8} z\right) \cap\left(x \oplus_{8} y\right) \oplus_{8} z$ for all $(y, z) \neq(D, D)$ and $C \in x \oplus_{8}\left(D \oplus_{8} D\right) \cap\left(x \oplus_{8} D\right) \oplus_{8} D$.

- If $x=C$ then $C \in x \oplus_{8}\left(y \oplus_{8} z\right) \cap\left(x \oplus_{8} y\right) \oplus_{8} z$.

- If $x=D$ and $(y, z) \neq(D, D)$ then $C \in x \oplus_{8}\left(y \oplus_{8} z\right) \cap\left(x \oplus_{8} y\right) \oplus_{8} z$ for all $(y, z) \neq(D, D)$.

Therefore, $\left(H, \oplus_{8}\right)$ is a commutative $H_{v}$-semigroup.

Let $H=\{A, B, C, D\}$, for all $x, y \in H$, we define " $\otimes_{8}$ " on $H$, where $x \otimes_{8} y=z$ and $z$ is the product of any spontaneous redox reaction that occurs between $x$ and $y$. Then we obtain the following tables for $\left(H, \otimes_{8}\right)$ :

\begin{tabular}{|c|c|c|c|c|}
\hline$\otimes_{8}$ & $A$ & $B$ & $C$ & $D$ \\
\hline$A$ & $A$ & $\{A, B\}$ & $\{A, C\}$ & $H$ \\
\hline$B$ & $\{A, B\}$ & $B$ & $\{B, C\}$ & $H$ \\
\hline$C$ & $\{A, C\}$ & $\{B, C\}$ & $C$ & $\{C, D\}$ \\
\hline$D$ & $H$ & $H$ & $\{C, D\}$ & $D$ \\
\hline
\end{tabular}

Proposition 3.5. $\left(\{A, B\}, \otimes_{8}\right),\left(\{A, C\}, \otimes_{8}\right),\left(\{B, C\}, \otimes_{8}\right),\left(\{C, D\}, \otimes_{8}\right)$, and $\left(\{A, B, C\}, \otimes_{8}\right)$ are hypergroups.

Proof. Since $\left(\{A, B\}, \otimes_{8}\right),\left(\{A, C\}, \otimes_{8}\right),\left(\{B, C\}, \otimes_{8}\right),\left(\{C, D\}, \otimes_{8}\right)$ and $\left(\{A, B, C\}, \otimes_{8}\right)$ are biset hypergroups, it follows that they are hypergroups. 
Remark 3.4. Every element in $\left(H, \otimes_{8}\right)$ is idempotent, that is $x \otimes_{8} x=x$.

Proposition 3.6. Let $H=\{A, B, C, D\}$. Then $\left(H, \otimes_{8}\right)$ is semihypergroup.

Proof. Since $\left(\{A, B\}, \otimes_{8}\right),\left(\{A, C\}, \otimes_{8}\right),\left(\{B, C\}, \otimes_{8}\right),\left(\{C, D\}, \otimes_{8}\right)$, and $\left(\{A, B, C\}, \otimes_{8}\right)$ are hypergroups, it suffices to consider the following cases for associativity in Table 3.5. Thus, $\left(H, \otimes_{8}\right)$ is semihypergroup.

Theorem 3.6. Let $H=\{A, B, C, D\}$. Then $\left(H, \otimes_{8}\right)$ is a commutative hypergroup.

Proof. The proof is followed by Propositions 3.5 and 3.6.

\subsection{Case $\alpha=\gamma>\beta$}

Let $H=\{A . B, C, D\}$, it's clear that:

$$
\beta<E_{1}<\alpha=\gamma, \beta<E_{2}<\alpha=\gamma, \beta<E_{3}<\gamma=\alpha
$$

The following are all possible spontaneous redox combinations of $H$. $A+A \longrightarrow A+A[0]$ $A+B \longrightarrow\left\{\begin{array}{c}B+A[0] \\ C+A\left[\gamma-E_{3}\right] \\ A+D\left[\gamma-E_{2}\right]\end{array}\right.$

$A+C \longrightarrow C+A[0]$

We have $E_{1}-E_{3}=\frac{\alpha n_{1}+\beta n_{2}}{n_{1}+n_{2}}-\frac{\beta n_{2}+\gamma n_{3}}{n_{2}+n_{3}}=n_{2}\left(n_{1}-n_{3}\right)(\alpha-\beta)$.

Case $n_{1}>n_{3}$. We get that

$$
A+D \longrightarrow\left\{\begin{array}{c}
C+C\left[\alpha-E_{3}\right] \\
C+B\left[E_{1}-E_{3}\right] \\
D+C\left[\alpha-E_{2}\right] \\
D+A[0]
\end{array}\right.
$$

Case $n_{1} \leq n_{3}$. We get that

$$
A+D \longrightarrow\left\{\begin{array}{c}
C+C\left[\alpha-E_{3}\right] \\
D+C\left[\alpha-E_{2}\right] \\
D+A[0]
\end{array}\right.
$$

$B+B \longrightarrow\left\{\begin{array}{c}B+B[0] \\ A+C[-\beta+\gamma] \\ A+D\left[\gamma-E_{1}\right]\end{array}\right.$
$B+C \longrightarrow\left\{\begin{array}{c}C+B[0] \\ C+A\left[\gamma-E_{3}\right]\end{array}\right.$ 
Table 3.5: Associativity of $\left(H, \otimes_{8}\right)$

\begin{tabular}{|c|c|}
\hline$D \otimes_{8}\left(A \otimes_{8} A\right)=D \otimes_{8} A=H$ & $\left(D \otimes_{8} A\right) \otimes_{8} A=H \otimes_{8} A=H$ \\
\hline$A \otimes_{8}\left(D \otimes_{8} A\right)=A \otimes_{8} H=H$ & $\left(A \otimes_{8} D\right) \otimes_{8} A=H \otimes_{8} A=H$ \\
\hline$A \otimes_{8}\left(A \otimes_{8} D\right)=A \otimes_{8} H=H$ & $\left(A \otimes_{8} A\right) \otimes_{8} D=A \otimes_{8} D=H$ \\
\hline$D \otimes_{8}\left(B \otimes_{8} B\right)=D \otimes_{8} B=H$ & $\left(D \otimes_{8} B\right) \otimes_{8} B=H \otimes_{8} B=H$ \\
\hline$B \otimes_{8}\left(D \otimes_{8} B\right)=B \otimes_{8} H=H$ & $\left(B \otimes_{8} D\right) \otimes_{8} B=H \otimes_{8} B=H$ \\
\hline$B \otimes_{8}\left(B \otimes_{8} D\right)=B \otimes_{8} H=H$ & $\left(B \otimes_{8} B\right) \otimes_{8} D=B \otimes_{8} D=H$ \\
\hline$D \otimes_{8}\left(D \otimes_{8} D\right)=D \otimes_{8} D=D$ & $\left(D \otimes_{8} D\right) \otimes_{8} D=D \otimes_{8} D=D$ \\
\hline$A \otimes_{8}\left(D \otimes_{8} D\right)=A \otimes_{8} D=H$ & $\left(A \otimes_{8} D\right) \otimes_{8} D=H \otimes_{8} D=H$ \\
\hline$B \otimes_{8}\left(D \otimes_{8} D\right)=B \otimes_{8} D=H$ & $\left(B \otimes_{8} D\right) \otimes_{8} D=H \otimes_{8} D=H$ \\
\hline$D \otimes_{8}\left(A \otimes_{8} D\right)=D \otimes_{8} H=H$ & $\left(D \otimes_{8} A\right) \otimes_{8} D=H \otimes_{8} D=H$ \\
\hline$D \otimes_{8}\left(B \otimes_{8} D\right)=D \otimes_{8} H=H$ & $\left(D \otimes_{8} B\right) \otimes_{8} D=H \otimes_{8} D=H$ \\
\hline$D \otimes_{8}\left(D \otimes_{8} A\right)=D \otimes_{8} H=H$ & $\left(D \otimes_{8} D\right) \otimes_{8} A=D \otimes_{8} A=H$ \\
\hline$D \otimes_{8}\left(D \otimes_{8} C\right)=D \otimes_{8}\{C, D\}=\{C, D\}$ & $\left(D \otimes_{8} D\right) \otimes_{8} C=D \otimes_{8} C=\{C, D\}$ \\
\hline$A \otimes_{8}\left(B \otimes_{8} D\right)=A \otimes_{8} H=H$ & $\left(A \otimes_{8} B\right) \otimes_{8} D=\{A, B\} \otimes_{8} D=H$ \\
\hline$A \otimes_{8}\left(C \otimes_{8} D\right)=A \otimes_{8}\{C, D\}=H$ & $\left(A \otimes_{8} C\right) \otimes_{8} D=\{A, C\} \otimes_{8} D=H$ \\
\hline$A \otimes_{8}\left(D \otimes_{8} B\right)=A \otimes_{8} H=H$ & $\left(A \otimes_{8} D\right) \otimes_{8} B=H \otimes_{8} B=H$ \\
\hline$A \otimes_{8}\left(D \otimes_{8} C\right)=A \otimes_{8}\{C, D\}=H$ & $\left(A \otimes_{8} D\right) \otimes_{8} C=H \otimes_{8} C=H$ \\
\hline$B \otimes_{8}\left(A \otimes_{8} D\right)=B \otimes_{8} H=H$ & $\left(B \otimes_{8} A\right) \otimes_{8} D=H \otimes_{8} D=H$ \\
\hline$B \otimes_{8}\left(C \otimes_{8} D\right)=B \otimes_{8}\{C, D\}=H$ & $\left(B \otimes_{8} C\right) \otimes_{8} D=\{B, C\} \otimes_{8} D=H$ \\
\hline$B \otimes_{8}\left(D \otimes_{8} A\right)=B \otimes_{8} H=H$ & $\left(B \otimes_{8} D\right) \otimes_{8} A=H \otimes_{8} A=H$ \\
\hline$B \otimes_{8}\left(D \otimes_{8} C\right)=B \otimes_{8}\{C, D\}=H$ & $\left(B \otimes_{8} D\right) \otimes_{8} C=H \otimes_{8} C=H$ \\
\hline$C \otimes_{8}\left(A \otimes_{8} D\right)=C \otimes_{8} H=H$ & $\left(C \otimes_{8} A\right) \otimes_{8} D=\{A, C\} \otimes_{8} D=H$ \\
\hline$C \otimes_{8}\left(B \otimes_{8} D\right)=C \otimes_{8} H=H$ & $\left(C \otimes_{8} B\right) \otimes_{8} D=\{B, C\} \otimes_{8} D=H$ \\
\hline$C \otimes_{8}\left(D \otimes_{8} A\right)=C \otimes_{8} H=H$ & $\left(C \otimes_{8} D\right) \otimes_{8} A=\{C, D\} \otimes_{8} A=H$ \\
\hline$C \otimes_{8}\left(D \otimes_{8} B\right)=C \otimes_{8} H=H$ & $\left(C \otimes_{8} D\right) \otimes_{8} B=\{C, D\} \otimes_{8} B=H$ \\
\hline$D \otimes_{8}\left(A \otimes_{8} B\right)=D \otimes_{8}\{A, B\}=H$ & $\left(D \otimes_{8} A\right) \otimes_{8} B=H \otimes_{8} B=H$ \\
\hline$D \otimes_{8}\left(A \otimes_{8} C\right)=D \otimes_{8}\{A, C\}=H$ & $\left(D \otimes_{8} A\right) \otimes_{8} C=H \otimes_{8} C=H$ \\
\hline$D \otimes_{8}\left(B \otimes_{8} A\right)=D \otimes_{8} H=H$ & $\left(D \otimes_{8} B\right) \otimes_{8} A=H \otimes_{8} A=H$ \\
\hline$D \otimes_{8}\left(B \otimes_{8} C\right)=D \otimes_{8}\{B, C\}=H$ & $\left(D \otimes_{8} B\right) \otimes_{8} C=H \otimes_{8} C=H$ \\
\hline$D \otimes_{8}\left(C \otimes_{8} A\right)=D \otimes_{8}\{A, C\}=H$ & $\left(D \otimes_{8} C\right) \otimes_{8} A=\{C, D\} \otimes_{8} A=H$ \\
\hline$D \otimes_{8}\left(C \otimes_{8} B\right)=D \otimes_{8}\{B, C\}=H$ & $\left(D \otimes_{8} C\right) \otimes_{8} B=\{C, D\} \otimes_{8} B=H$ \\
\hline
\end{tabular}




$$
\begin{aligned}
& B+D \longrightarrow\left\{\begin{array}{c}
C+C[-\beta+\alpha] \\
C+B\left[-\beta+E_{1}\right] \\
C+A\left[-\beta+E_{2}\right] \\
D+C\left[\alpha-E_{1}\right] \\
D+B[0]
\end{array}\right. \\
& C+C \longrightarrow C+C[0] \\
& C+D \longrightarrow D+C[0] \\
& D+D \longrightarrow D+D[0]
\end{aligned}
$$

For all $x, y \in H$, we define " $\oplus_{9}$ " on $H$ for the case $n_{1}>n_{3}$ and the case $n_{1} \leq n_{3}$, where $x \oplus_{9} y=z$, where $z$ is the major product. We obtain the table below for $\left(H, \oplus_{9}\right)$ :

\begin{tabular}{|c|c|c|c|c|}
\hline$\oplus_{9}$ & $A$ & $B$ & $C$ & $D$ \\
\hline$A$ & $A$ & $\{A, C\}$ & $\{A, C\}$ & $C$ \\
\hline$B$ & $\{A, C\}$ & $\{A, C\}$ & $\{A, C\}$ & $C$ \\
\hline$C$ & $\{A, C\}$ & $\{A, C\}$ & $C$ & $\{C, D\}$ \\
\hline$D$ & $C$ & $C$ & $\{C, D\}$ & $D$ \\
\hline
\end{tabular}

Theorem 3.7. $\left(H, \oplus_{9}\right)$ is a commutative $H_{v}$-semigroup.

Proof. $\left(H, \oplus_{9}\right)$ is isomorphic to $\left(H, \oplus_{1}\right)$ in Theorem 3.2.

Let $H=\{A, B, C, D\}$, for all $x, y \in H$, we define " $\otimes_{9}$ " on $H$ for the case $n_{1}>n_{3}$, " $\otimes_{10}$ " for the case $n_{1} \leq n_{3}$, where $x \otimes_{9} y=z$ where $z$ is the product of any spontaneous redox reaction that occurs between $x$ and $y$. Then we obtain the tables for $\left(H, \otimes_{9}\right)$ and $\left(H, \otimes_{10}\right)$ :

\begin{tabular}{|c|c|c|c|c|}
\hline$\otimes_{9}$ & $A$ & $B$ & $C$ & $D$ \\
\hline$A$ & $A$ & $H$ & $\{A, C\}$ & $H$ \\
\hline$B$ & $H$ & $H$ & $\{A, B, C\}$ & $H$ \\
\hline$C$ & $\{A, C\}$ & $\{A, B, C\}$ & $C$ & $\{C, D\}$ \\
\hline$D$ & $H$ & $H$ & $\{C, D\}$ & $D$ \\
\hline
\end{tabular}

Proposition 3.7. $\left(H, \otimes_{9}\right)$ is a commutative hypergroup.

Proof. $\left(H, \otimes_{9}\right)$ is isomorphic to $\left(H, \otimes_{1}\right)$ in Theorem 3.3.

Proposition 3.8. $\left(H, \otimes_{10}\right)$ is a commutative hypergroup. 


\begin{tabular}{|c|c|c|c|c|}
\hline$\otimes_{10}$ & $A$ & $B$ & $C$ & $D$ \\
\hline$A$ & $A$ & $H$ & $\{A, C\}$ & $\{A, C, D\}$ \\
\hline$B$ & $H$ & $H$ & $\{A, B, C\}$ & $H$ \\
\hline$C$ & $\{A, C\}$ & $\{A, B, C\}$ & $C$ & $\{C, D\}$ \\
\hline$D$ & $\{A, C, D\}$ & $H$ & $\{C, D\}$ & $D$ \\
\hline
\end{tabular}

Proof. It is clear that $\left(H, \otimes_{10}\right)$ is a quasi-hypergroup since all elements of $H$ are present in every row and column, so it remains to prove that $\left(H, \otimes_{10}\right)$ is a semihypergroup, i.e. associative.

Since $\left(\{a, c\}, \otimes_{10}\right)$ and $\left(\{a, c, d\}, \otimes_{10}\right)$ are associative, then it suffices to consider the cases for associativity in a similar way that is done in Table 3.5 .

Remark 3.5. In some of the above spontaneous reactions, not all spontaneous reactions are considered because their presence or absence does not affect our results.

\section{Examples of Chemical hyperstructures}

In this section, we present some elements with four oxidation states and identify their chemical hyperstructure. In particular, we present Copper and Astatine as an examples under the case $\alpha>\gamma>\beta$ with $E_{1}>\gamma$ and $E_{2}>\gamma$, Iridium under the case $\alpha>\gamma=\beta$ with $E_{1}, E_{2}>\gamma$, and Uranium under the case $\alpha>\gamma>\beta$ with $E_{1}>\gamma$ and $E_{2}>\gamma$.

Example 4.1. Copper, denoted as $C u$, is a soft and ductile element with very high thermal and electrical conductivity. Copper is one of the few metals that can occur in nature in a directly usable metallic form native metals. The Latimer diagram of Copper in acid solution satisfying the condition $\alpha \geq \gamma \geq \beta$ is given as follows:

$$
\mathrm{CuO}^{+} \longrightarrow{ }_{1.8} \mathrm{Cu}^{2+} \longrightarrow_{0.159} \mathrm{Cu}^{+} \longrightarrow 0.521 \mathrm{Cu} .
$$

Copper has four different oxidation states: $+3,+2,+1$, and 0 . We denote $C u O^{+}$by $C u^{3+}$. We have $\alpha=1.8, \beta=0.159, \gamma=0.521$ and $n_{1}=n_{2}=n_{3}=1$. Since $E_{1}=$ $\frac{(1.8)(1)+(0.159)(1)}{2}=0.979>\gamma$, and $E_{2}=\frac{(1.8)(1)+(0.159)(1)+(0.521)(1)}{3}=0.826>\gamma$, it follows that:

- $\left(H=\left\{C u, C u^{+}, C u^{2+}, C u^{3+}\right\}, \oplus_{2}\right)$ is a commutative $H_{v^{-} \text {semigroup. }}$

According to Table 4.1, $C u^{2+}$ is the most common oxidation state.

- $\left(H=\left\{C u, C u^{+}, C u^{2+}, C u^{3+}\right\}, \otimes_{2}\right)$ is a commutative hypergroup with the following table:

Every element $e$ in $H$ is identity since for all $x \in H, x \in x \otimes e \cap e \otimes x$.

Example 4.2. [2] Astatine denoted as $A t$ is a radioactive chemical element and it is the heaviest known halogen. The Latimer diagram of Astatine in base solution satisfying the condition $\alpha>\gamma>\beta$ is given as follows: 
Table 4.1: $\left(\left\{C u, C u^{+}, C u^{2+}, C u^{3+}\right\}, \oplus_{2}\right)$

\begin{tabular}{|c|c|c|c|c|}
\hline$\oplus_{2}$ & $C u$ & $C u^{+}$ & $C u^{2+}$ & $C u^{3+}$ \\
\hline$C u$ & $C u$ & $\left\{C u, C u^{2+}\right\}$ & $\left\{C u, C u^{2+}\right\}$ & $C u^{2+}$ \\
\hline$C u^{+}$ & $\left\{C u, C u^{2+}\right\}$ & $\left\{C u, C u^{2+}\right\}$ & $\left\{C u, C u^{2+}\right\}$ & $C u^{2+}$ \\
\hline$C u^{2+}$ & $\left\{C u, C u^{2+}\right\}$ & $\left\{C u, C u^{2+}\right\}$ & $C u^{2+}$ & $\left\{C u^{2+}, C u^{3+}\right\}$ \\
\hline$C u^{3+}$ & $C u^{2+}$ & $C u^{2+}$ & $\left\{C u^{2+}, C u^{3+}\right\}$ & $C u^{3+}$ \\
\hline
\end{tabular}

Table 4.2: $\left(\left\{C u, C u^{+}, C u^{2+}, C u^{3+}\right\}, \otimes_{2}\right)$

\begin{tabular}{|c|c|c|c|c|}
\hline$\otimes_{2}$ & $C u$ & $C u^{+}$ & $C u^{2+}$ & $C u^{3+}$ \\
\hline$C u$ & $C u$ & $\left\{C u, C u^{+}, C u^{2+}\right\}$ & $\left\{C u, C u^{2+}\right\}$ & $H$ \\
\hline$C u^{+}$ & $\left\{C u, C u^{+}, C u^{2+}\right\}$ & $\left\{C u, C u^{+}, C u^{2+}\right\}$ & $\left\{C u, C u^{+}, C u^{2+}\right\}$ & $H$ \\
\hline$C u^{2+}$ & $\left\{C u, C u^{2+}\right\}$ & $\left\{C u, C u^{+}, C u^{2+}\right\}$ & $C u^{2+}$ & $\left\{C u^{2+}, C u^{3+}\right\}$ \\
\hline$C u^{3+}$ & $H$ & $H$ & $\left\{C u^{2+}, C u^{3+}\right\}$ & $C u^{3+}$ \\
\hline
\end{tabular}

$$
A t^{5+} \longrightarrow_{0.5} A t^{+} \longrightarrow_{0} A t_{2} \longrightarrow_{0.2} A t^{-} .
$$

Al-Tahan et al. in [2], studied the $H_{v}$-semigroup and hypergroup associated to Astatine. Their results on Astatine can be also concluded from our results of Section 3.

Astatine has four oxidation states: $+5,+1,0,-1$. We have $\alpha=0.5, \beta=0, \gamma=$ 0.2 and $n_{1}=4, n_{2}=1, n_{3}=1$. Since $E_{1}=\frac{(0.5)(4)+(0)(1)}{5}=0.4>\gamma$ and $E_{2}=$ $\frac{(0.5)(4)+(0)(1)+(0.2)(1)}{6}=0.367>\gamma$, we get the following results:

- $\left(H=\left\{\stackrel{6}{A} t^{-}, A t_{2}, A t^{+}, A t^{5+}\right\}, \oplus_{2}\right)$ is a commutative $H_{v}$-semigroup.

Table 4.3: $\left(\left\{A t^{-}, A t_{2}, A t^{+}, A t^{5+}\right\}, \oplus_{2}\right)$

\begin{tabular}{|c|c|c|c|c|}
\hline$\oplus_{2}$ & $A t^{-}$ & $A t_{2}$ & $A t^{+}$ & $A t^{5+}$ \\
\hline$A t^{-}$ & $A t^{-}$ & $\left\{A t^{-}, A t^{+}\right\}$ & $\left\{A t^{-}, A t^{+}\right\}$ & $A t^{5+}$ \\
\hline$A t_{2}$ & $\left\{A t^{-}, A t^{+}\right\}$ & $\left\{A t^{-}, A t^{+}\right\}$ & $\left\{A t^{-}, A t^{+}\right\}$ & $A t^{+}$ \\
\hline$A t^{+}$ & $\left\{A t^{-}, A t^{+}\right\}$ & $\left\{A t^{-}, A t^{+}\right\}$ & $A t^{+}$ & $\left\{A t^{+}, A t^{5+}\right\}$ \\
\hline$A t^{5+}$ & $A t^{+}$ & $A t^{+}$ & $\left\{A t^{+}, A t^{5+}\right\}$ & $A t^{5+}$ \\
\hline
\end{tabular}

According to Table 4.3, $A t^{+}$is the most common oxidation state.

- $\left(H=\left\{A t^{-}, A t_{2}, A t^{+}, A t^{5+}\right\}, \otimes_{2}\right)$ is a commutative $H_{v}-$ semigroup.

According to Table $4.4, A t^{5+}$ is the least common oxidation state.

Example 4.3. Uranium (U) is a metallic, silver-gray element that is a member of the actinide series. It is the principle fuel for nuclear reactors, but it also used in the manufacture of nuclear weapons. The Latimer diagram of Uranium in base solution satisfying the condition $\alpha>\gamma>\beta$ is given as follows: 
Table 4.4: $\left(\left\{A t^{-}, A t_{2}, A t^{+}, A t^{5+}\right\}, \otimes_{2}\right)$

\begin{tabular}{|c|c|c|c|c|}
\hline$\otimes_{2}$ & $A t^{-}$ & $A t_{2}$ & $A t^{+}$ & $A t^{5+}$ \\
\hline$A t^{-}$ & $A t^{-}$ & $\left\{A t^{-}, A t_{2}, A t^{+}\right\}$ & $\left\{A t^{-}, A t^{+}\right\}$ & $H$ \\
\hline$A t_{2}$ & $\left\{A t^{-}, A t_{2}, A t^{+}\right\}$ & $\left\{A t^{-}, A t_{2}, A t^{+}\right\}$ & $\left\{A t^{-}, A t_{2}, A t^{+}\right\}$ & $H$ \\
\hline$A t^{+}$ & $\left\{A t^{-}, A t^{+}\right\}$ & $\left\{A t^{-}, A t_{2}, A t^{+}\right\}$ & $A t^{+}$ & $\left\{A t^{+}, A t^{5+}\right\}$ \\
\hline$A t^{5+}$ & $H$ & $H$ & $\left\{A t^{+}, A t^{5+}\right\}$ & $A t^{5+}$ \\
\hline
\end{tabular}

$$
U^{6+} \longrightarrow_{-0.3} U^{4+} \longrightarrow_{-2.6} U^{3+} \longrightarrow_{-2.1} U .
$$

Uranium has four oxidation states: $+6,+4,+3,0$. We have $\alpha=-0.3, \beta=-2.6, \gamma=$ -2.1 . Since $E_{1}=\frac{(-0.3)(2)+(-2.6)(1)}{3}=-1.06>\gamma$ and $E_{2}=\frac{(-0.3)(2)+(-2.6)(1)+(3)(-2.1)}{6}=$ $-1.58>\gamma$. We get the following results:

- $\left(H=\left\{U, U^{3+}, U^{4+}, U^{6+}\right\}, \oplus_{2}\right)$ is a commutative $H_{v}$-semigroup.

Table 4.5: $\left(\left\{U, U^{3+}, U^{4+}, U^{6+}\right\}, \oplus_{2}\right)$

\begin{tabular}{|c|c|c|c|c|}
\hline$\oplus_{2}$ & $U$ & $U^{3+}$ & $U^{4+}$ & $U^{6+}$ \\
\hline$U$ & $U$ & $\left\{U, U^{4+}\right\}$ & $\left\{U, U^{4+}\right\}$ & $U^{4+}$ \\
\hline$U^{3+}$ & $\left\{U, U^{4+}\right\}$ & $\left\{U, U^{4+}\right\}$ & $\left\{U, U^{4+}\right\}$ & $U^{4+}$ \\
\hline$U^{4+}$ & $\left\{U, U^{4+}\right\}$ & $\left\{U, U^{4+}\right\}$ & $U^{4+}$ & $\left\{U^{4+}, U^{6+}\right\}$ \\
\hline$U^{6+}$ & $U^{4+}$ & $U^{4+}$ & $\left\{U^{4+}, U^{6+}\right\}$ & $U^{6+}$ \\
\hline
\end{tabular}

According to Table 4.5, $U^{4+}$ is the most common oxidation state.

- $\left(H=\left\{U, U^{3+}, U^{4+}, U^{6+}\right\}, \otimes_{2}\right)$ is a hypergroup with the following table.

Table 4.6: $\left(\left\{U, U^{3+}, U^{4+}, U^{6+}\right\}, \otimes_{2}\right)$

\begin{tabular}{|c|c|c|c|c|}
\hline$\otimes_{2}$ & $U$ & $U^{3+}$ & $U^{4+}$ & $U^{6+}$ \\
\hline$U$ & $U$ & $\left\{U, U^{3+}, U^{4+}\right\}$ & $\left\{U, U^{4+}\right\}$ & $H$ \\
\hline$U^{3+}$ & $\left\{U, U^{3+}, U^{4+}\right\}$ & $\left\{U, U^{3+}, U^{4+}\right\}$ & $\left\{U, U^{3+}, U^{4+}\right\}$ & $H$ \\
\hline$U^{4+}$ & $\left\{U, U^{4+}\right\}$ & $\left\{U, U^{3+}, U^{4+}\right\}$ & $U^{4+}$ & $\left\{U^{4+}, U^{6+}\right\}$ \\
\hline$U^{6+}$ & $H$ & $H$ & $\left\{U^{4+}, U^{6+}\right\}$ & $U^{6+}$ \\
\hline
\end{tabular}

According to Table $4.6, U^{6+}$ is the least common oxidation state.

Example 4.4. Iridium (Ir) is a chemical element, a very hard, brittle, silvery-white transition metal of the platinum group. The Latimer diagram of Iridium in base solution satisfying the condition $\alpha>\gamma=\beta$ is given as follows:

$$
\mathrm{Ir}^{6+} \longrightarrow 0.4 \mathrm{Ir}^{4+} \longrightarrow_{0.1} \mathrm{Ir}^{3+} \longrightarrow_{0.1} \mathrm{Ir} .
$$


Iridium has four oxidation states $+6,+4,+3,0$. We have $\alpha=0.4, \beta=\gamma=0.1$ and $n_{1}=$ $2, n_{2}=1, n_{3}=3$. Since $E_{1}=\frac{(0.4)(2)+(0.1)(1)}{3}=0.3>\gamma$ and $E_{2}=\frac{(0.4)(2)+(0.1)(1)+(0.1)(3)}{6}=$ $0.2>\gamma$, we get the following results:

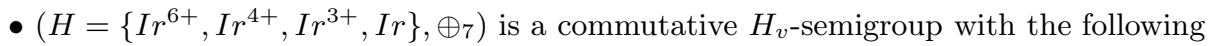
table:

Table 4.7: $\left(\left\{\operatorname{Ir}^{6+}, \operatorname{Ir}{ }^{4+}, \operatorname{Ir}{ }^{3+}, \operatorname{Ir}\right\}, \oplus_{7}\right)$

\begin{tabular}{|c|c|c|c|c|}
\hline$\oplus_{7}$ & $I r$ & $I r^{3+}$ & $I r^{4+}$ & $I r^{6+}$ \\
\hline$I r$ & $I r$ & $\left\{I r, I r^{3+}\right\}$ & $\left\{I r, I r^{4+}\right\}$ & $\left\{I r^{3+}, I r^{4+}\right\}$ \\
\hline$I r^{3+}$ & $\left\{I r, I r^{3+}\right\}$ & $I r^{3+}$ & $\left\{I r^{3+}, I r^{4+}\right\}$ & $I r^{4+}$ \\
\hline$I r^{4+}$ & $\left\{I r, I r^{4+}\right\}$ & $\left\{I r^{3+}, I r^{4+}\right\}$ & $I r^{4+}$ & $\left\{I r^{4+}, I r^{6+}\right\}$ \\
\hline$I r^{6+}$ & $\left\{I r^{3+}, I r^{4+}\right\}$ & $I r^{4+}$ & $\left\{I r^{4+}, I r^{6+}\right\}$ & $I r^{6+}$ \\
\hline
\end{tabular}

According to above table $4.7, I^{6+}$ is the least common oxidation state. - $\left(H=\left\{I r^{6+}, I r^{4+}, I r^{3+}, I\right\}, \otimes_{7}\right)$ is a commutative hypergroup.

Table 4.8: $\left(\left\{I r^{6+}, I r^{4+}, I r^{3+}, I r\right\}, \otimes_{7}\right)$

\begin{tabular}{|c|c|c|c|c|}
\hline$\otimes_{7}$ & $I r$ & $\operatorname{Ir}^{3+}$ & $\operatorname{Ir}^{4+}$ & $\operatorname{Ir}^{6+}$ \\
\hline$I r$ & $I r$ & $\left\{I r, I r^{3+}\right\}$ & $\left\{I r, I r^{4+}\right\}$ & $H$ \\
\hline$I r^{3+}$ & $\left\{I r, I r^{3+},\right\}$ & $\left\{I r^{3+}\right\}$ & $\left\{I r^{3+}, I^{4+}\right\}$ & $H$ \\
\hline$I r^{4+}$ & $\left\{I r, I r^{4+}\right\}$ & $\left\{I r^{3+}, I r^{4+}\right\}$ & $I r^{4+}$ & $\left\{I r^{4+}, I r^{6+}\right\}$ \\
\hline$I r^{6+}$ & $H$ & $\mathrm{H}$ & $\left\{I r^{4+}, I r^{6+}\right\}$ & $I r^{6+}$ \\
\hline
\end{tabular}

According to Table 4.8, $\mathrm{Ir}^{4+}$ is the most common oxidation state.

\section{Conclusion}

This paper dealt with non-isomorphic ( $H_{v}$-semigroups) hypergroups of an element with four oxidation states which has the largest number of examples among all cases for elements with four oxidation states.

For future work, it will be interesting to generalize our work to arbitrary elements with $k$ - oxidation states.

\section{REF E R E N C E S}

1. M. Al- Tahan, B. Davvaz, Weak chemical hyperstructures associated to electrochemical cells, Iranian Journal of Mathematical Chemistry, 9(1)(2018): 65-75. 
2. M. Al- Tahan, B. Davvaz, Chemical hyperstructures for Astatine, Tellurium and for Bismuth, Bull. Comput. Math., 7(1)(2019) 9-25.

3. M. Al- Tahan, B. Davvaz, Chemical hyperstructures for elements with four oxidation states, (submitted).

4. K.M. Chun, Chemical hyperstructures of chemical reactions for Titanium, Journal of the Chungcheong Mathematical Society, 30(4) (2017).

5. S.-C. Chung, K.M. Chun, N.J. Kim, S.Y. Jeong, H. Sim, J. Lee and H. Maeng, Chemical hyperalgebras for three oxidation states of elements, MATCH Commun. Math. Comput. Chem., 72(2) (2014), 389-402.

6. P. Corsini, Prolegomena of Hypergroup Theory. Udine, Tricesimo, Italy: Second edition, Aviani editore, 1993.

7. P. Corsini and V. Leoreanu, Applications of Hyperstructures Theory, Advances in Mathematics, Kluwer Academic Publisher, 2003.

8. B. Davvaz, Polygroup Theory and Related Systems, World Scientific Publishing Co. Pte. Ltd., Hackensack, NJ, 2013. viii+200 pp.

9. B. Davvaz, Semihypergroup Theory, Elsevier/Academic Press, London, 2016. viii+156 pp.

10. B. Davvaz, Weak algebraic hyperstructures as a model for interpretation of chemical reactions, Iranian Journal of Mathematical Chemistry, 7 (2) (2016) 267-283.

11. B. Davvaz and A. Dehgan Nezhad, Chemical examples in hypergroups, Ratio Matematica, 14 (2003), 71-74.

12. B. Davvaz, A brief survey of the theory of $H_{v}$-structures, Proc. 8th International Congress on Algebraic Hyperstructures and Applications, 1-9 Sep., 2002, Samothraki, Hreece, Spanidis Press, (2003) 39-70.

13. B. Davvaz, A. Dehghan Nezhad and A. Benvidi, Chemical hyperalgebra: Dismutation reactions, MATCH Commun. Math. Comput. Chem., 67 (2012), 55-63.

14. B. Davvaz, A. Dehghan Nezhad and A. Benvidi, Chain reactions as experimental examples of ternary algebraic hyperstructures, MATCH Commun. Math. Comput. Chem., 65(2) (2011), 491-499.

15. B. Davvaz, A. Dehghan Nezhad and M. Mazloum-Ardakani, Chemical hyperalgebra: Redox reactions, MATCH Commun. Math. Comput. Chem., 71 (2014), 323-331.

16. B. Davvaz and V. Leoreanu-Fotea, Hyperring Theory and Applications, International Academic Press, USA, 2007.

17. B. Douglas, D. H. McDaniel, and J. J. Alexander, Concepts and Models of Inorganic Chemistry, 2nd ed. John Wiely \& Sons, Inc. 1985.

18. F. Marty, Sur une generalization de la notion de group, In: 8th Congress Math. Scandenaves, (1934), pp. 45-49.

19. T. Vougiouklis, $H_{v}$-groups defined on the same set, Discrete Math., 155 (1996) 259265.

20. T. Vougiouklis, Hyperstructures and Their Representations, Hadronic Press, Inc, 115, Palm Harber, USA, 1994.

21. T. Vougiouklis, The fundamental relation in hyperrings. The general hyperfield, In: Proc. Fourth Int. Congress on Algebraic Hyperstructures and Appl. (AHA 1990), World Scientific, 1991, pp. 203-211. 
Rawia Al-Jinani

Lebanese International University

Faculty of Science

Department of Mathematics and Physics

P. O. Box 5 Jeb-jenine

Khyara, Lebanon

21430322@students.liu.edu.1b

Madeleine Al-Tahan

Lebanese International University

Faculty of Science

Department of Mathematics and Physics

P. O. Box 5 Jeb-jenine

Khyara, Lebanon

madeline.tahan@liu.edu.lb

Bijan Davvaz

Yazd University

Faculty of Science

Department of Mathematics

P. O. Box 89195-741

Yazd, Iran

davvaz@yazd.ac.ir 